OPEN ACCESS

Edited by:

Paolo Montuschi

Catholic University of the Sacred

Heart, Italy

Reviewed by:

Vaidehi Thanawala,

Vir Biotechnology, Inc., United States

Antonio Molino,

University of Naples Federico II, Italy

*Correspondence:

Xiaobo Huang

659492700@qq.com

Rongan Liu

frog007_119@qq.com

${ }^{\text {t}}$ These authors have contributed equally to this work

Specialty section:

This article was submitted to Respiratory Pharmacology,

a section of the journal

Frontiers in Pharmacology

Received: 18 March 2020

Accepted: 21 August 2020

Published: 15 October 2020

Citation:

Yang F, Yue R, Luo X, Liu R and Huang $X$ (2020) Hydrogen:

A Potential New Adjuvant Therapy for COVID-19 Patients.

Front. Pharmacol. 11:543718. doi: 10.3389/fphar.2020.543718

\section{Hydrogen: A Potential New Adjuvant Therapy for COVID-19 Patients}

\author{
Fuxun Yang ${ }^{\dagger}$, Ruiming Yue ${ }^{\dagger}$, Xiaoxiu Luo, Rongan Liu ${ }^{*}$ and Xiaobo Huang ${ }^{*}$ \\ Department of Critical Care Medicine, Sichuan Provincial People's Hospital, Chengdu, China
}

Hydrogen has been shown to have antioxidant, anti-inflammatory, hormone-regulating, and apoptosis-resistance properties, among others. Based on a review of the research, the use of hydrogen might reduce the destructive cytokine storm and lung injury caused by SARS-CoV-2 during COVID-19 (Corona Virus Disease 2019) in the early stage, stimulating ropy sputum drainage, and ultimately reducing the incidence of severe disease. Molecular hydrogen treatment has the potential to become a new adjuvant therapy for COVID-19, but its efficacy and safety require large clinical trials and further confirmation.

Keywords: Hydrogen, COVID-19, therapy, cytokine storm, mucus

\section{INTRODUCTION}

Since the Corona Virus Disease 2019 (COVID-19) was first reported in Wuhan at the end of December 2019, it quickly became the sixth-largest public health emergency and a matter of international concern (Lai et al., 2020). Up to 11:00 on July 31, 2020, there were 17,328,002 confirmed cases in the world, and the cumulative number of deaths was 670,287 with an overall mortality rate of $3.8 \%$ (https://covid19.who.int/). Moreover, there is no specific antiviral drug or vaccine that could be used to prevent COVID-19. Huang et al. (2020) found that the plasma concentrations of IL-2, IL-7, IL-10, and TNF- $\alpha$ in severe or critical patients were higher than those in other patients. This is consistent with the pathological findings of Wang Fushen (Liu et al., 2020; Xu Z. et al., 2020). Therefore, Chen et al. propose that cytokine storm is one of the most important factors of the disease in critically ill patients (Chen et al., 2020). Currently, there is no specific drug that can be used to treat cytokine storms.

Hydrogen is a colorless, odorless, and tasteless gas. In 2007, Ohsawa et al. (2007) published a paper in Nature outlining that the inhalation of $2 \%$ hydrogen could selectively eliminate hydroxyl radical $(\mathrm{OH})$ and peroxynitrite anion (ONOO-) and significantly improve cerebral ischemiareperfusion injury in rats, which has initiated an upsurge in molecular biology research based on hydrogen. To date, the biological effects of hydrogen have been widely studied. Based on its biological effects, such as in anti-oxidation, anti-inflammation, anti-apoptosis, and hormone regulatory, it has been established that hydrogen has protective effects against a variety of diseases. In particular, the small molecular properties of hydrogen ensure that it quickly reaches the alveoli, which suggests a unique advantage for lung diseases. Given the current epidemic and based on clinical experience, safety, operability, and simple clinical promotion, this review discusses the feasibility of hydrogen as a means of controlling and preventing COVID-19. 


\section{HYDROGEN AND THE CYTOKINE STORM}

Immune cells can become activated, producing pro-inflammatory cytokines, including tumor necrosis factor- $\alpha$ (TNF- $\alpha$ ), interleukins (such as IL-1 $\beta$ and IL-6), and interferon- $\gamma$ (IFN- $\gamma$ ) (Taniguchi and Karin, 2018). An effect of cytokines is the activation of the NADPH oxidase in leukocytes, which leads to the production of reactive oxygen species (ROS) such as superoxide, hydroxyl radicals, and singlet oxygen (Liu et al., 2015). In 1993, Ferrara et al. first proposed the concept of a cytokine storm in graft-versus-host disease (Ferrara et al., 1993). SARS coronavirus infection was found to induce an interferon- $\gamma$ related cytokine storm, which might be related to the immunopathological damage observed in SARS patients (Huang et al., 2005). In 2005, a study on avian influenza A H5N1 suggested that high viral loads and the resulting intense inflammatory response are key to its onset (de Jong et al., 2006). Cytokine storms have also been reported in influenza (Kalil and Thomas, 2019) and Middle East respiratory syndrome (MERS) (Channappanavar and Perlman, 2017). At present, the factor that causes cytokine storms is not clear, but it is generally believed that the immune system overreacts to new and highly pathogenic pathogens. The relating imbalance of the immune regulatory network, the lack of negative feedback, and the continuous self-amplification of positive feedback lead to an abnormal increase in many kinds of cytokines, and finally to a cytokine storm. Although the pathophysiological mechanism underlying COVID-19 is not completely understood, it has been reported that there are large quantities of cytokines such as IL-1 $\beta$, INF- $\gamma$, IP-10, and MCP-1 in COVID-19 patients, which might activate Th1 cells. The concentrations of G-CSF, IP-10, MCP-1, MIP-IA, and TNF- $\alpha$ in critically ill patients were found to be higher than those in non-critical patients, indicating that cytokine storms might be related to the severity of the disease (Liu et al., 2020). The effectiveness of anti-IL6-receptor and glucocorticoid therapy for patients with COVID-19 was only verified in a small number of patients (Selvaraj et al., 2020; Xu X. et al., 2020). However, more clinical studies are underway with respect to treating COVID-19 with tocilizumab and dexamethasone (NCT04445272, NCT04244591, NCT04381364). Corticosteroids suppress lung inflammation but also inhibit immune responses and pathogen clearance (Russell et al., 2020). Furthermore, the use of anti-IL6-receptor therapy for patients with rheumatic diseases might lead to an increased risk of infection (Rutherford et al., 2018). Due to these potential side effects, tocilizumab and dexamethasone have not been widely used in clinical practice.

Cytokines released excessively can cause acute lung injury in patients. An increase in TNF- $\alpha$ levels will lead to the activation of inflammatory cytokines such as IL-1, IL-6, and IL-8 (Chen et al., 2015). At the same time, high mobility group box1 (HMGB1) (Ma et al., 2015), CCL2 (Hillman et al., 2007), and Egr-1 (Hoetzel et al., 2008) all affect the release of inflammatory factors. Keliang Xie found that hydrogen can suppress the infiltration of neutrophils and macrophages in lung tissue, inhibit the activity of NF- $\mathrm{KB}$ and MPO in lung tissue, and reduce inflammatory factors and cytokine secretion in lung tissue, including TNF- $\alpha$, IL-1, IL-6, and HMGB1. Hydrogen can eliminate ROS, such as hydroxyl and peroxynitrate anions, while maintaining the normal metabolism of redox reactions and other ROS (Xie et al., 2012). Accordingly, hydrogen treatment can reduce the levels of TNF- $\alpha$, IL-1, IL-1 $\beta$, IL-6, IL-8, HMGB1, CCL2, and Egr-1 in lung tissue in an animal model (Huang et al., 2010a). Furthermore, inhaling hydrogen for 45 minutes can reduce airway inflammation in patients with asthma and COPD (Wang et al., 2020). At the same time, previous studies have shown that an increase in IL-10 can inhibit the synthesis and release of inflammatory cells and colony stimulating factors (Laveda et al., 2006). After inhaling hydrogen, IL-10 was found to increase in the serum and sputum supernatant of sanitation workers (Gong et al. 2016), indicating that this treatment can affect anti-inflammatory reactions and reduce secondary injury caused by cytokine storms. Some critical patients with pneumonia need to be supported by mechanical ventilation. However, this can cause lung injury or aggravate the original lung injury. In a rat model of mechanically ventilated lung injury, Huang et al. (Huang et al., 2010a) found that after inhaling $2 \%$ hydrogen the expression of NF-kappa B was activated, promoting the expression of the antiapoptotic protein Bcl-2, inhibiting expression of the apoptotic protein Bax, suppressing inflammatory factor expression, decreasing the lung histopathological score, and alleviating pulmonary edema, thus diminishing ventilator-related acute lung injury. In addition, hydrogen can inhibit the Rho/ROCK pathway, increase the expression of ZO-1, and protect lung tissue cells by improving cell-to-cell permeability, and reducing lung injury (Zhang et al., 2016). Therefore, the early use of hydrogen in COVID-19 patients could potentially suppress the release of cytokines and reduce lung injury.

\section{HYDROGEN AND OXIDATIVE STRESS REACTIONS IN COVID-19}

Superoxide dismutase (SOD) is an important antioxidant enzyme in the antioxidant defense system of the body. It can remove a variety of toxic or oxidizing substances in the body to eliminate the damage to DNA and biological functional proteins caused by these substances to maintain the stability of the internal environment and contribute to anti-toxicity and anti-oxidation processes (Gwarzo and Muhammad, 2010). After hydrogen treatment, the content of malondialdehyde in lung tissue can be reduced, increasing the activity of SOD (Shi et al. 2013). This helps to maintain the stability of the internal environment of the body to achieve excessive activation of oxidative processes and reduce the oxidative stress caused by the ROS pathway. Multiple organ failure is a common cause of death in critically ill COVID-19 patients. Hydrogen may be used to protect multiple organs including the heart, kidney, and nervous system via anti-apoptotic and anti-oxidative functions to maintain the normal response of the body and reduce mortality (Hayashida et al., 2012; Hayashida et al., 2014; Homma et al., 2014). 


\section{HYDROGEN REDUCES COVID-19- RELATED VISCOUS SECRETIONS}

Based on the results of pathological anatomy, Liu Liang's team found that in addition to excessive inflammatory reactions, many viscous secretions that spilled from the alveoli and fibrous cords could be seen in lung sections, and the viscous secretions were mainly concentrated in the terminal bronchi (Liu et al., 2020). This is inconsistent with the clinical manifestation of dry cough without sputum. Clinical oxygen therapy is delivered mainly via nasal high-flow oxygen inhalation and non-invasive ventilatorassisted ventilation. Accordingly, its positive pressure ventilation mode will cause the accumulation of distal bronchial viscous secretions, increase airway resistance, alter the effect of oxygen therapy, and aggravate systemic hypoxia. This finding implies new thinking that could adjust the regimen used in clinical treatment. Drug atomization and humidification might become indispensable treatment methods, but in terms of the treatment process, attention should be paid to the third-level protection of medical staff to prevent aerosol transmission, which would increase the risk of infection. Mucus is composed of water, ions, lipids, proteins, and complexes (Voynow et al., 2006). In an animal model, airway mucus was found to play an important role in the host defense mechanism, but the production of excessive mucus is harmful (Shimizu et al., 2012). Muc5ac and Muc5b are the components of mucin, and Muc5ac is produced by goblet cells among airway epithelial cells (Perezvilar et al., 2006). Rats treated with hydrogen-enriched water had reduced airway damage, Muc5ac expression, and mucus secretion in smog-induced COPD models (Ning et al., 2013). Therefore, early hydrogen inhalation may promote sputum dilution, improve small airway resistance, and relieve dyspnea.

\section{SAFETY OF HYDROGEN}

Based on a clinical hydrogen test, hydrogen absorption and drinking hydrogen-rich water are used for treatment. The potential anti-fatigue and performance benefits of hydrogen rich water (HRW) have received increased research interest over the past 5 years. For example, acute pre-exercise supplementation with HRW reduces blood lactate at higher exercise intensities, improves the exercise-induced perception of effort, and increases ventilatory efficiency (Botek et al. 2019). At the same time, hydrogen, as a flammable and explosive small molecular substance, has been developed clinically and can be safely applied with access to medical devices. Clinical research has shown that hydrogen dissolved in irrigation solution reduces corneal endothelial damage during phacoemulsification (Igarashi et al., 2019).

\section{REFERENCES}

Atsunori, N., Yoshiya, T., Prachi, S., Malkanthi, E., and Najla, G. (2010). Effectiveness of Hydrogen Rich Water on Antioxidant Status of Subjects with Potential Metabolic Syndrome-An Open Label Pilot Study. J. Clin. Biochem. Nutr. 46 (2), 140-149. doi: 10.3164/jcbn.09-100
Furthermore, breathing $\mathrm{H}_{2}-\mathrm{O}_{2}$ might reduce the inspiratory effort in patients with acute severe tracheal stenosis and can be used safely for this purpose (Zhou et al., 2019). Although $\mathrm{H}_{2}$ gas is flammable, concentrations $<4 \%$, together with oxygen at room temperature, are incombustible. As indicated by the second law of thermodynamics, although several physical processes that satisfy the first law are possible, the only processes that occur in nature are those for which the entropy of the system either remains constant or increases. Thus, exhaled $\mathrm{H}_{2}$ diffuses instantly, without accumulating or resulting in an increased concentration exceeding the inspiratory $\mathrm{H}_{2}$ concentration. Therefore, $2 \% \mathrm{H}_{2}$ gas can be carefully administered in a hospital (Tamura et al., 2017). During this treatment, a very small number of patients have symptoms such as sparse stool, increased defecation frequency, heartburn, and headache after drinking. These symptoms may be relieved without intervention after the patient stops hydrogen treatment. At the same time, these symptoms have no serious adverse events, and the associated harm is minimal (Atsunori et al., 2010; Huang et al., 2010b).

\section{CONCLUSIONS}

In summary, we hypothesize that the early use of hydrogen might mitigate the destruction caused by the cytokine storm associated with COVID-19, reducing lung injury, promoting viscous sputum drainage, and thus reducing the incidence of critically ill patients. Only one other article to date has mentioned the use of hydrogen to treat COVID-19 patients (Guan et al., 2020). In the future, more large-scale randomized controlled trials are needed to verify the efficacy and safety of this treatment clinically.

\section{AUTHOR CONTRIBUTIONS}

FX Y, RM Y, and RA L wrote the original draft. XL undertook validation, writing, review, and editing. $\mathrm{XH}$ undertook writing, review, and editing. All authors contributed to the article and approved the submitted version.

\section{FUNDNG}

This study was supported by the Sichuan Province Science and Technology Support Program (numbers: 2017SZ0138)and Chengdu Science and Technology Support Program (numbers: 2020-YF05-00104-SN).

Botek, M., Krejčí, J., McKune, A. J., Sládečková, B., and Naumovski, N. (2019). Hydrogen Rich Water Improved Ventilatory, Perceptual and Lactate Responses to Exercise. Int. J. Sports Med. 40 (14), 879-885. doi: 10.1055/a-0991-0268

Channappanavar, R., and Perlman, S. (2017). Pathogenic human coronavirus infections: causes and consequences of cytokine storm and immunopathology. Semin. Immunopathol. 39, 529-539. doi: 10.1007/s00281-017-0629-x 
Chen, X., Liu, Q., Wang, D., Feng, S., Zhao, Y., Shi, Y., et al. (2015). Protective Effects of Hydrogen-Rich Saline on Rats with Smoke Inhalation Injury. Oxid. Med. Cell Longev. 2015, 106836. doi: 10.1155/2015/106836

Chen, N., Zhou, M., Dong, X., Qu, J., Gong, F., Han, Y., et al. (2020). Epidemiological and clinical characteristics of 99 cases of 2019 novel coronavirus pneumonia in Wuhan, China: a descriptive study. Lancet 395 (10223), 507-513. doi: 10.1016/S0140-6736(20)30211-7

de Jong, M. D., Simmons, C. P., Thanh, T. T., Hien, V. M., Smith, G. J., Chau, T. N., et al. (2006). Fatal outcome of human influenza A (H5N1) is associated with high viral load and hypercytokinemia. Nat. Med. 12 (10), 1203-1207. doi: $10.1038 / \mathrm{nm} 1477$

Ferrara, J. L. M., Abhyankar, S., and Gilliland, D. G. (1993). Cytokine storm of graft-versus-host disease: A critical effector role for interleukin-1. Transplant. Proc. 25 (1 Pt 2), 1216-1217.

Gong, Z. J., Guan, J. T., Ren, X. Z., Meng, D. Y., and Yan, X. X. (2016). Protective effect of hydrogen on the lung of sanitation workers exposed to haze. Chinese Journal of Tuberculosis and Respiratory Diseases. 3912 (12), 916-923. doi: 10.3760/cma.j.issn.1001-0939.2016.12.003

Guan, W. J., Wei, C. H., Chen, A. L., Sun, X. C., Guo, G. Y., Zou, X., et al. (2020). Hydrogen/oxygen mixed gas inhalation improves disease severity and dyspnea in patients with Coronavirus disease 2019 in a recent multicenter, open-label clinical trial. J. Thorac. Dis. 12 (6), 3448-3452. doi: $10.21037 /$ jtd-2020-057

Gwarzo, M. Y., and Muhammad, A. K. (2010). Extracellular Superoxide Dismutase Activity and Plasma Malondialdehyde in Human Immunodeficiency Virus Subjects of Kano State as Surrogate Markers of CD4 Status. Int. J. Biomed. Sci. Ijbs 6 (4), 294-300.

Hayashida, K., Sano, M., Kamimura, N., Yokota, T., Suzuki, M., Maekawa, Y., et al. (2012). H2 Gas Improves Functional Outcome After Cardiac Arrest to an Extent Comparable to Therapeutic Hypothermia in a Rat Model. J. Am. Heart Assoc. 1 (5), e003459-e003459. doi: 10.1161/JAHA.112.003459

Hayashida, K., Sano, M., Kamimura, N., Yokota, T., Suzuki, M., Ohta, S., et al. (2014). Hydrogen Inhalation During Normoxic Resuscitation Improves Neurological Outcome in a Rat Model of Cardiac Arrest Independently of Targeted Temperature Management. Circulation 132 (24), 2173-2180. doi: 10.1161/CIRCULATIONAHA.114.011848

Hillman, N. H., Moss, T. J., Kallapur, S. G., Bachurski, C., Pillow, J. J., Polglase, G. R., et al. (2007). Brief, large tidal volume ventilation initiates lung injury and a systemic response in fetal sheep. Am. J. Respir. Crit. Care Med. 176 (6), 575581. doi: 10.1164/rccm.200701-051OC

Hoetzel, A., Dolinay, T., Vallbracht, S., Zhang, Y., Kim, H. P., Ifedigbo, E., et al. (2008). Carbon monoxide protects against ventilator-induced lung injury via PPAR-gamma and inhibition of Egr-1. Am. J. Respir. Crit. Care Med. 177 (11), 1223-1232. doi: 10.1164/rccm.200708-1265OC

Homma, K., Yoshida, T., Yamashita, M., Hayashida, K., Hayashi, M., and Hori, S. (2014). Inhalation of Hydrogen Gas Is Beneficial for Preventing ContrastInduced Acute Kidney Injury in Rats. Nephron Exp. Nephrol. 128 (3-4), 116122. doi: $10.1159 / 000369068$

Huang, K. J., Su, I. J., Theron, M., Wu, Y. C., Lai, S. K., Liu, C. C., et al. (2005). An interferon-gamma-related cytokine storm in SARS patients. J. Med. Virol. 75 (2), 185-194. doi: 10.1002/jmv.20255

Huang, C. S., Kawamura, T., Lee, S., Tochigi, N., Shigemura, N., Buchholz, B. M., et al. (2010a). Hydrogen inhalation ameliorates ventilator-induced lung injury. Crit. Care 14 (6), R234. doi: 10.1186/cc9389

Huang, C. S., Kawamura, T., Toyoda, Y., and Nakao, A. (2010b). Recent Advances in Hydrogen Research as a Therapeutic Medical Gas. Free Radic. Res. 44 (9), 971-982. doi: 10.3109/10715762.2010.500328

Huang, C., Wang, Y., Li, X., Ren, L., Zhao, J., Hu, Y., et al. (2020). Clinical features of patients infected with 2019 novel coronavirus in Wuhan, China. Lancet 395 (10223), 497-506. doi: 10.1016/S0140-6736(20)30183-5

Igarashi, T., Ohsawa, I., Kobayashi, M., Umemoto, Y., Arima, T., Suzuki, H., et al. (2019). Effects of Hydrogen in Prevention of Corneal Endothelial Damage During Phacoemulsification: A Prospective Randomized Clinical Trial. Am. J. Ophthalmol. 207, 10-17. doi: 10.1016/j.ajo.2019.04.014

Kalil, A. C., and Thomas, P. G. (2019). Influenza virus-related critical illness: pathophysiology and epidemiology. Crit. Care 23 (1), 1-7. doi: 10.1186/ s13054-019-2539-x
Lai, C.-C., Shih, T.-P., Ko, W.-C., Tang, H.-J., and Hsueh, P.-R. (2020). Severe acute respiratory syndrome coronavirus 2 (SARS-CoV-2) and coronavirus disease-2019 (COVID-19): The epidemic and the challenges. Int. J. Antimicrobial. Agents 55 (3), 1-9. doi: 10.1016/j.ijantimicag.2020.105924

Laveda, R., Martinez, J., Munoz, C., Penalva, J. C., and Perez-Mateo, M. (2006). Different profile of cytokine synthesis according to the severity of acute pancreatitis. World J. Gastroenterol. 11 (34), 5309-5313. doi: 10.3748/wjg.v11.i34.5309

Liu, Q., Zhou, Y. H., and Yang, Z. Q. (2015). The cytokine storm of severe influenza and development of immunomodulatory therapy. Cell. Mol. Immunol. 13 (1), 3-10. doi: 10.1038/cmi.2015.74

Liu, Q., Wang, R. S., Qu, G. Q., Wang, Y. Y., and Liu, L. (2020). Gross examination report of a COVID-19 death autopsy. Fa Yi Xue Za Zhi 36 (1), 21-23. doi: 10.12116/j.issn.1004-5619.2020.01.005

Ma, L., Zeng, J., Mo, B., Wang, C., Huang, J., Sun, Y., et al. (2015). High mobility group box 1: a novel mediator of Th2-type response-induced airway inflammation of acute allergic asthma. J. Thorac. Dis. 7 (10), 1732-1741. doi: 10.3978/j.issn.2072-1439.2015.10.18

Ning, Y., Yan, S., Huang, H., Zhang, J., Dong, Y., Xu, W., et al. (2013). Attenuation of Cigarette Smoke-Induced Airway Mucus Production by Hydrogen-Rich Saline in Rats. PLoS One 8 (12), e83429. doi: 10.1371/ journal.pone.0083429

Ohsawa, I., Ishikawa, M., Takahashi, K., Watanabe, M., Nishimaki, K., Yamagata, K., et al. (2007). Hydrogen acts as a therapeutic antioxidant by selectively reducing cytotoxic oxygen radicals. Nat. Med. 13 (6), 688-694. doi: 10.1038/nm1577

Perezvilar, J., Mabolo, R., Mcvaugh, C. T., Bertozzi, C. R., and Boucher, R. C. (2006). Mucin Granule Intraluminal Organization in Living Mucous/Goblet Cells ROLES OF PROTEIN POST-TRANSLATIONAL MODIFICATIONS AND SECRETION. J. Biol. Chem. 281 (8), 4844. doi: 10.1074/ jbc.M510520200

Russell, C. D., Millar, J. E., and Baillie, J. K. (2020). Clinical evidence does not support corticosteroid treatment for 2019-nCoV lung injury. Lancet 395 (10223), 473-475. doi: 10.1016/S0140-6736(20)30317-2

Rutherford, A.III., Subesinghe, S., Hyrich, K. L., and Galloway, J. (2018). Serious infection across biologic-treated patients with rheumatoid arthritis: results from the British Society for Rheumatology Biologics Register for Rheumatoid Arthritis. Ann. Rheumatic Dis. A. J. Clin. Rheumatol. Connect. Tissue Res. 77 (6), 905-910. doi: 10.1136/annrheumdis-2017-212825

Selvaraj, V, Dapaah-Afriyie, K, and Finn, A. (2020). Short-Term Dexamethasone in Sars-CoV-2 Patients. Rhode Island Med. J. 103 (6), 39-43.

Shi, H. M., Zhou, H. C., Jia, Y. R., Wang, Y., and Liu, J. F. (2013). The effect of hydrogen on hemorrhagic shock induced acute lung injury in rats. Zhonghua Wei Zhong Bing Ji Jiu Yi Xue 25 (6), 347-350. doi: 10.3760/cma.j.issn.20954352.2013.06.008

Shimizu, T., Hirano, H., Shimizu, S., Kishioka, C., Sakakura, Y., and Majima, Y. (2012). Differential properties of mucous glycoproteins in rat nasal epithelium. A comparison between allergic inflammation and lipopolysaccharide stimulation. Am. J. Respir. Crit. Care Med. 164 (6), 1077-1082. doi: 10.1164/ ajrccm.164.6.2012058

Tamura, T., Hayashida, K., Sano, M., Onuki, S., and Suzuki, M. (2017). Efficacy of inhaled HYdrogen on neurological outcome following BRain Ischemia During post-cardiac arrest care (HYBRID II trial): study protocol for a randomized controlled trial. Trials 18 (1), 488. doi: 10.1186/s13063-017-2246-3

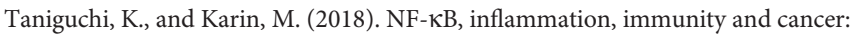
coming of age. Nat. Rev. Immunol. 185 (5), 309-324. doi: 10.1038/nri.2017.142

Voynow, J. A., Gendler, S. J., and Rose, M. C. (2006). Regulation of mucin genes in chronic inflammatory airway diseases. Am. J. Respir. Cell Mol. Biol. 34 (6), 661665. doi: 10.1165/rcmb.2006-0035SF

Wang, S.-T., Bao, C., He, Y., Tian, X., Yang, Y., Zhang, T., et al. (2020). Hydrogen gas (XEN) inhalation ameliorates airway inflammation in asthma and COPD patients. QJM Monthly J. Assoc. Physicians, hcaa:164. doi: 10.1093/qjmed/hcaal64

Xie, K., Yu, Y., Yi, H., Zheng, L., Li, J., Chen, H., et al. (2012). Molecular Hydrogen Ameliorates Lipopolysaccharide-induced Acute Lung Injury in Mice through Reducing Inflammation and Apoptosis. Shock 37 (5), 548-555. doi: 10.1097/ SHK.0b013e31824ddc81

Xu, X., Han, M., Li, T., Sun, W., and Wei, H. (2020). Effective treatment of severe COVID-19 patients with tocilizumab. Proc. Natl. Acad. Ences 8 (4), 420-422. doi: $10.1073 /$ pnas.2005615117 
Xu, Z., Shi, L., Wang, Y., Zhang, J., Huang, L., Zhang, C., et al. (2020). Pathological findings of COVID-19 associated with acute respiratory distress syndrome. Lancet Respirat. Med. 8 (4), 420-422. doi: 10.1016/S2213-2600(20)30076-X

Zhang, H., Liu, L., Yu, Y., Sun, Z., Liang, Y., and Yu, Y. (2016). [Role of Rho/ROCK signaling pathway in the protective effects of hydrogen against acute lung injury in septic mice]. Zhonghua Wei Zhong Bing Ji Jiи Yi Xue 28 (5), 401-406. doi: 10.3760/cma.j.issn.2095-4352.2016.05.005

Zhou, Z. Q., Zhong, C. H., Su, Z. Q., Li, X. Y., Chen, Y., Chen, X. B., et al. (2019). Breathing Hydrogen-Oxygen Mixture Decreases Inspiratory Effort in Patients with Tracheal Stenosis. Respiration 97 (1), 42-51. doi: 10.1159/000492031
Conflict of Interest: The authors declare that the research was conducted in the absence of any commercial or financial relationships that could be construed as a potential conflict of interest.

Copyright $\odot 2020$ Yang, Yue, Luo, Liu and Huang. This is an open-access article distributed under the terms of the Creative Commons Attribution License (CC BY). The use, distribution or reproduction in other forums is permitted, provided the original author(s) and the copyright owner(s) are credited and that the original publication in this journal is cited, in accordance with accepted academic practice. No use, distribution or reproduction is permitted which does not comply with these terms. 